
\title{
$\angle S$ Research Square \\ Using outbreak data to estimate the dynamic COVID-19 landscape in Eastern Africa
}

\author{
Mark Wamalwa ( $\nabla$ mwamalwa@icipe.org ) \\ International Centre of Insect Physiology and Ecology \\ Henri E.Z. Tonnang \\ International Centre of Insect Physiology and Ecology
}

\section{Research Article}

Keywords: COVID-19, eSIR model, Runge-Kutta approximation, Basic reproduction number, Epidemic trend

Posted Date: August 26th, 2021

DOl: https://doi.org/10.21203/rs.3.rs-839877/v1

License: (c) (i) This work is licensed under a Creative Commons Attribution 4.0 International License. Read Full License 
available at the end of the article

Title: Using outbreak data to estimate the dynamic COVID-19 landscape in Eastern Africa

Mark Wamalwa* ${ }^{* 1,2}$, Henri E.Z. Tonnang ${ }^{1}$

${ }^{1}$ International Centre of Insect Physiology and Ecology (icipe), P.O. Box 30772-00100, Nairobi, Kenya,

*Corresponding author email address: mwamalwa@icipe.org

\section{Abstract}

\section{Background}

The emergence of Coronavirus disease 2019 (COVID-19) as a global pandemic presents a serious health threat to African countries and the livelihoods of its people. To mitigate the impact of this disease, these countries implemented intervention measures including self-isolation, the closure of schools, banning of public gatherings, social distancing and border closures. Several epidemiological models have been used to improve our understanding of COVID-19 trajectory. This has helped inform decisions about pandemic planning, resource allocation, implementation of other non-pharmaceutical interventions (NPIs). This study presents estimates of the cases and fatalities due to COVID-19 and attempts to forecast the impact of governmental interventions in Burundi, Ethiopia, Kenya, Rwanda, South Sudan, Tanzania and Uganda. .

\section{Methods}

We used time series COVID-19 case and mortality data collated from the Johns Hopkins University (JHU) repository and an extended susceptible-infected-removed (eSIR) compartmental model incorporating quarantine and vaccination compartments to account for transmission dynamics and vaccine-induced immunity over time. The predication accuracy was evaluated using the root mean square error and mean absolute error.

\section{Results}

The number of new and confirmed cases show an exponential trend since March 02 2020. The mean basic reproductive number $\left(\mathrm{R}_{0}\right)$ was between $1.32(95 \% \mathrm{CI}, 1.17$ - 1.49) in Rwanda and 8.52 (95\% CI: 3.73 14.10) in Kenya, under exponential growth. There would be a total of 115,505 (95\% CI:109,999 - 121,264), $7,072,584(6,945,505-7,203,084), 18,248,566(18,100,299-18,391,438), 410,599$ (399,776 - 421528), 386,020 (376,478 - 396244), 107,265 (95,757 - 119982), 3,145,602 (3,089,070 - 3205017) infected cases under the current country blockade by January 16/2022 in Burundi, Ethiopia, Kenya, Rwanda, South Sudan, Tanzania and Uganda respectively. We show that the low apparent morbidity and mortality observed in EACs, is likely biased by underestimation of infected and mortality cases.

\section{Conclusion}

The current NPI measures can effectively reduce further spread of COVID-19 and should be strengthened. The observed reduction in $\mathrm{R}_{0}$ is consistent with intervention measures implemented in EACs, in particular, lockdowns and roll-out of vaccination programmes. Future work should account for the negative impact of the interventions to the economy and food systems.

Keywords: COVID-19; eSIR model; Runge-Kutta approximation; Basic reproduction number; Epidemic trend. 


\section{Introduction}

Coronavirus Disease 2019 (COVID-19) is a zoonotic disease caused by the Severe Acute Respiratory

Syndrome Corona Virus 2 (SARS-CoV-2), a pathogen that was first discovered in Wuhan, China in 2019.

Consequently, the disease has spread all over the world, leading to high morbidity and mortality in addition

to negatively impacting the healthcare systems and the economy [1,2]. Following the first case reported on the African continent on the 14th February, 2020 in Egypt, a total of 6,543,882 COVID-19 confirmed cases

Several non-pharmaceutical interventions (NPIs) have been implemented across EACs to contain the coronavirus disease (COVID-19) pandemic. Social distancing (SD) interventions applied so far have included school closures, remote working and quarantine [5]. While serological testing, contact tracing, physical distancing, hand hygiene, masks, and quarantine measures may reduce transmission, many countries have resorted to lockdown measures with varying degree of success [6-8]. Previously, these measures have been shown to have large impacts on pandemic influenza transmission [9]. However, the time point of implementation of NPIs is key to their success in reducing the peak of the epidemic [10]. As the pandemic evolves, it is imperative that the impact of NPIs is quantified in terms of their efficacy and appropriate use in order to influence public health policy. These measures need to be appropriately justified to the population in terms of their public perception and the optimal time when they could be eased [11]. 
Indeed, mathematical models have been used to unravel the understanding of coronavirus disease 2019

(COVID-19), the disease caused by SARS-CoV-2 virus $[5,12,13]$. The essential components in analyzing

all dynamic processes that contribute to disease transmission, include, first, solving the mathematical model

numerically (forward modeling) and second, estimating the unknown parameters in the model (inverse

modeling) [14]. Recently, the scientific community has used mathematical models to understand the

COVID-19 disease transmission dynamics, including the impact of NPIs. For example, the University of

Washington, Seattle, developed the Institute of Health Metrics (IHME) for fitting parametrized curves to

COVID-19 data using extendable nonlinear mixed effects model [15]. The Imperial College London (ICL),

developed a semi-mechanistic Bayesian model to estimate the rate of transmission, total number of infected people and mortality at a given time point, and the impact of NPIs on the basic reproduction number $\left(\mathrm{R}_{0}\right)$ as well as the time-varying reproduction number $\mathrm{R}(\mathrm{t})$, [5]. The basic reproduction number $\left(\mathrm{R}_{0}\right)$, a measure

Compartmental standard SIR models, eSIR model and the more complex Susceptible-Exposed-InfectiousRecovered (SEIR) use ordinary differential equations and a three- or four-state Markov chain to solve ordinary differential equations that mimic the infectious disease trajectory [24]. The eSIR model assumes a constant the transmission rate in the SIR model. However, the transmission rate can be altered to mimic 
02 NPIs, such as personal protective measures, quarantine, and city lockdown. Indeed, the eSIR model 03 introduces a transmission modifier $\pi(\mathrm{t})$ to the SIR model, to allow a time-varying probability of the 04 transmission rate (Figure 1).

Figure 1. The extended Susceptible-Exposed-Removed (eSIR) basic model. The transmission rate modifier $\pi(\mathrm{t})$ takes on values according to actual interventions in different countries [22].

The eSIR model assumes that probabilities of the three compartments follow a Markov transition process with input as the proportions of infected and removed (recovered and dead) cases. The observed proportions of infected and removed cases on day $\mathrm{t}$ are denoted by $Y_{\mathrm{t}}{ }^{I}$ and $Y_{\mathrm{t}}{ }^{R}$, respectively. The true underlying

(Equation 2)

$$
\begin{aligned}
& \frac{d \theta_{t}^{S}}{d t}=-\beta \pi(t) \theta_{t}^{S} \theta_{t}^{I} \\
& \frac{d \theta_{t}^{I}}{d t}=-\beta \pi(t) \theta_{t}^{S} \theta_{t}^{I}-\gamma \theta_{t}^{I}, \\
& \frac{d \theta_{t}^{R}}{d t}=\gamma \theta_{t}^{I}
\end{aligned}
$$

probabilities of the $\mathrm{S}, \mathrm{I}$, and $\mathrm{R}$ compartments on day $\mathrm{t}$ are denoted by $\theta_{\mathrm{t}}^{S}, \theta_{\mathrm{t}}{ }^{I}$, and $\theta_{\mathrm{t}}{ }^{R}$, respectively, and assume that for any $\mathrm{t}, \theta_{\mathrm{t}}{ }^{S}+\theta_{\mathrm{t}}{ }^{I}+\theta_{\mathrm{t}}^{R}=1$, which can be solved through ordinary differential equations (Equation 1-3):

Whereby, $\beta>0$ is the disease transmission rate, and $\gamma>0$ is the removal rate. $R_{0}=\beta / \gamma$ is the basic reproduction number assuming the whole population is susceptible. The Markov chain Monte Carlo (MCMC) algorithm is used to implement this model and provide the posterior estimates and credible intervals of the unknown parameters, $\mathrm{R}_{0}, \beta$, and $\gamma[17,22]$. 


\section{Materials and Methods}

\section{Data Sources}

28 We used publicly available COVID-19 daily recorded time-series data for the seven EACs (Burundi, 29 Ethiopia, Kenya, Rwanda, South Sudan, United Republic of Tanzania, and Uganda) from WHO and the 30 Johns Hopkins University Center for Systems Science and Engineering (JHU CCSE). This dataset includes daily counts of confirmed cases, recovered cases, and deaths from 22 January 2020.

\section{Epidemiological modelling}

34 Modelling of the impact of NPIs was implemented in R (version 4.0.4) and the differential equations were solved via the fourth-order Runge-Kutta approximation $[25,26]$. The input data was daily counts of confirmed cases and removed (recovered and deaths) cases. To estimate the epidemic trend in seven EACs, the year 2021 as the training dataset while the remainder was used for testing the model. To avoid overfitting, the data was divided into a training set (70\%) and testing set (30\%). We first trained the model on the daily confirmed cases ("training set"), and then used $30 \%$ of data from model predictions ("testing set") to gauge the accuracy of the resulting model [27]. The $\mathrm{R}_{0}$ was estimated using MCMC algorithm implemented in RJAGS and presented using the resulting posterior mean and $95 \%$ credible interval $(\mathrm{CI})$ [28]. The basic SIR model does not consider NPIs in the projection of epidemic trajectories, hence we sort to use time-varying transmission (tvt) rate model, SIR with time-varying quarantine, antibody (herd immunity) and vaccination compartments to project future scenario(s).

\section{SIR model with a time-varying transmission rate}

The following parameters were used to run the time-varying transmission (tvt) rate model: the transition rate modifier, $\pi(\mathrm{t})$, was allowed to vary between $(1.0,0.9,0.5$, and 0.1$)$ according to actual governmental interventions. This was set at $\pi(\mathrm{t})=0.95$ if $\mathrm{t}<\operatorname{Mar} 10$, for city lockdown; $\pi(\mathrm{t})=0.9$ if $\mathrm{t} \in(\operatorname{Mar} 10$, Mar 22), 
country lockdown; $\pi(\mathrm{t})=0.5$ if $\mathrm{t} \in($ Mar 15, April 01), shutdown of schools and non-essential businesses;

$52 \pi(\mathrm{t})=0.1$ if $\mathrm{t}>\operatorname{Mar} 31$, which corresponds to more enhanced quarantine protocols [23]. MCMC simulation 53 was performed using four parallel chains, with the number of draws in each chain, $M=5 \mathrm{e} 5$ and a burn-in 54 period of nburnin $=2 \mathrm{e} 3$ under $2 \times 10^{5}$ iteration number of adaptation in the MCMC $\left(\right.$ nadapt $\left.=2 \times 10^{5}\right)[22]$. 55 A time-dependent rate parameter $(\pi(\mathrm{t}))$ was introduced to vary the transmission rate $(\beta)$ and gamma $(\gamma)$ the

\section{SIR with time-varying quarantine}

We simulated the impact of quarantine measure by including a fourth compartment of quarantine population $[22,23]$. This time-varying quarantine model was simulated using parameters described in the tvt rate model described above. A vector phi $(\phi)$ that assumes a Dirac delta function (a point mass prior at $0.1-0.4$ ) was used to alter transition from susceptible to the quarantine compartment at time points corresponding to the days when quarantine measures were imposed in each country [18].

\section{Herd immunity}

To simulate the presence of natural acquired immunity/antibodies against COVID-19 within the population, an antibody (A) compartment was introduced and thereby altering the eSIR to eSAIR model [23,29]. The antibody (A) compartment consists of infected (I) but recovered/self-immunized individuals, with rate constants determining transition between the four compartments of Susceptible, Antibody, Infected and Removed (SAIR). The model was run using time-varying transmission rate parameters described above 
under the assumption that about $20 \%(\alpha=0.2)$ of the susceptible population had acquired neutralizing

antibodies against SARS-CoV-2 (Equation 4).

$$
\frac{d \theta_{t}^{A}}{d t}=\alpha(t) \theta_{t}^{S}
$$

(Equation 4)

The probability of having neutralizing antibodies against COVID-19 was denoted by theta $\left(d \theta_{t}^{A}\right)$ at time point $t$, where $\alpha(t)$ is the rate constant for the proportion of people moved into the antibody (A) compartment from the susceptible compartment.

\section{Vaccination}

The impact of vaccination was simulated using the eSVIR model and specific time points when vaccination began in the EACs. Consequently, the vaccination (V) compartment was introduced to integrate vaccination data into the basic SIR model. The parameters used to run the SIR model with a time-varying transmission rate parameters described above under the assumption that about $2 \%(\alpha=0.02)$ of the susceptible population were vaccinated. However, Tanzania only began their vaccination campaign in July 2021, while Burundi is yet to receive vaccines. The differential equations to simulate vaccination is illustrated below (Equation 5).

$$
\frac{d \theta_{t}^{V}}{d t}=\alpha(t) \theta_{t}^{S}, \frac{d \theta_{t}^{S}}{d t}=-\alpha(t) \theta_{t}^{S}-\beta \pi(t) \theta_{t}^{S} \theta_{t}^{I}, \frac{d \theta_{t}^{I}}{d t}=\beta \pi(t) \theta_{t}^{S} \theta_{t}^{I}-\gamma \theta_{t}^{I} \text {, and } \frac{d \theta_{t}^{R}}{d t}=\gamma \theta_{t}^{I}
$$

(Equation 5)

\section{Validation of the model}

The reliability and usefulness of our approach, was evaluated by comparing the model predictions of COVID-19 case-counts against the observed data between 06/16/20 and 04/11/2021 in Ethiopia, Kenya, Rwanda and Uganda using two metrics, the Root Mean square error (RMSE), and Mean Absolute Error (MAE) [29]. The input data was divided into training set (70\%) and testing set (30\%) to avoid overfitting [27]. The model was calibrated using observed data of confirmed cases ("training set") starting from the date of implementation of the intervention up to $7 / 14$ days prior to the peak. Thereafter, model predictions 
:00 ("testing set") of case-counts after the training period were then compared with the observed trends to

:01 evaluate the prediction accuracy.

\section{:03 Results}

\section{:04 Scenario analysis of COVID-19 epidemic development}

:05 Simulation of NPIs estimated the effectiveness of government intervention in curbing the spread of the :06 disease. The number of new and confirmed cases in the seven EACs show an exponential increase since :07 March 02 to April 01 2020/2021 and 2021/2022. We estimated the posterior values of the mean basic :08 reproductive number $\left(\mathrm{R}_{0}\right)$ for COVID-19 to between 2.70 - 3.10 and $1.32-8.52$ under exponential growth :09 model for the time period of 2020/2021 and 2021/2022 (Table 1). The $\mathrm{R}_{0}$ posterior values and endpoint in :10 EACs for 2020/2021 and 2021/2022 window provide the epidemiological trends of the disease. infected cases in Burundi, Ethiopia, Kenya, Rwanda, South Sudan, United Republic of Tanzania, and Uganda respectively by January 16/2022 (Table 2). This number is alarming but it includes missed cases, asymptomatic and confirmed cases. 
Table 1. Estimated $\mathrm{R}_{0}$ and endpoint in EACs using the eSIR model for the year 2020/2021.

\begin{tabular}{|c|c|c|c|c|c|c|c|}
\hline & & R0 & & Endpoint & & $95 \% \mathrm{CI}$ & \\
\hline Country & Median & Mean & $95 \%$ CI & Mean & Date & Infected $^{1}$ & Removed $^{2}$ \\
\hline Burundi & 2.58 & 2.71 & $1.48-4.58$ & $05 / 02 / 20$ & $04 / 05 / 20-07 / 31 / 20$ & $\begin{array}{c}998 \\
(116-2884)\end{array}$ & $\begin{array}{c}351 \\
(41-1100)\end{array}$ \\
\hline Ethiopia & 2.62 & 2.75 & $1.57-4.65$ & $04 / 27 / 20$ & $04 / 04 / 20-07 / 01 / 20$ & $\begin{array}{c}6,566 \\
(474-24130)\end{array}$ & $\begin{array}{c}5,754 \\
(665-20408)\end{array}$ \\
\hline Kenya & 2.57 & 2.70 & $1.54-4.67$ & $04 / 26 / 20$ & $04 / 05 / 20-06 / 23 / 20$ & $\begin{array}{c}2,572 \\
(455-6876)\end{array}$ & $\begin{array}{c}2,317 \\
(263-7475) \\
\end{array}$ \\
\hline Rwanda & 2.96 & 3.10 & $3.10-5.22$ & $05 / 07 / 20$ & $04 / 08 / 20-07 / 27 / 20$ & $\begin{array}{c}964 \\
(259-2121)\end{array}$ & $\begin{array}{c}397 \\
(41-1370)\end{array}$ \\
\hline South Sudan & 2.60 & 2.71 & $2.71-4.59$ & $05 / 21 / 20$ & $04 / 16 / 20-09 / 17 / 20$ & $\begin{array}{c}2,171 \\
(130-10107)\end{array}$ & $\begin{array}{c}631 \\
(89-1920) \\
\end{array}$ \\
\hline Tanzania & 2.69 & 2.82 & $2.82-4.90$ & $05 / 01 / 20$ & $04 / 05 / 20-07 / 16 / 20$ & $\begin{array}{c}4,369 \\
(614-14483)\end{array}$ & $\begin{array}{c}3,353 \\
(428-11942)\end{array}$ \\
\hline Uganda & 2.75 & 2.87 & $2.87-4.79$ & $05 / 06 / 20$ & $04 / 06 / 20-08 / 03 / 20$ & $\begin{array}{c}4,219 \\
(648-12354)\end{array}$ & $\begin{array}{c}2,180 \\
(211-8107)\end{array}$ \\
\hline
\end{tabular}

${ }^{1}$ Means of predicted infected population at the endpoint followed by the confidence interval in brackets $(\alpha=0.05)$.

${ }^{2}$ Means of predicted removed (recovered + deaths) population at the endpoint followed by the confidence interval in brackets $(\alpha=0.05)$.

Table 2. Estimated $R_{0}$ and endpoint in EACs using the eSIR model for the year 2021/2022.

\begin{tabular}{|c|c|c|c|c|c|c|c|}
\hline & \multicolumn{3}{|c|}{ R0 } & \multirow{2}{*}{$\begin{array}{l}\text { Endpoint } \\
\text { Mean }\end{array}$} & \multicolumn{3}{|c|}{$95 \%$ CI } \\
\hline Country & Median & Mean & $95 \%$ CI & & Date & Infected $^{1}$ & Removed $^{2}$ \\
\hline Burundi & 2.74 & 2.84 & $1.83-4.45$ & $01 / 16 / 22$ & $01 / 16 / 22$ & $\begin{array}{c}115,505 \\
(109,999-121,264)\end{array}$ & $\begin{array}{c}153,638 \\
(147508- \\
159954)\end{array}$ \\
\hline Ethiopia & 1.63 & 1.64 & $1.39-1.99$ & $01 / 16 / 22$ & $01 / 16 / 22$ & $\begin{array}{c}7,072,584 \\
(6,945,505-7,203,084)\end{array}$ & $\begin{array}{c}19,736,568 \\
(19521417- \\
19952888)\end{array}$ \\
\hline Kenya & 8.39 & 8.52 & $3.73-14.10$ & $01 / 16 / 22$ & $01 / 16 / 22$ & $\begin{array}{c}330,562 \\
(307493-353404)\end{array}$ & $\begin{array}{c}18,248,566 \\
(18,100,299- \\
18,391,438)\end{array}$ \\
\hline Rwanda & 1.31 & 1.32 & $1.17-1.49$ & $01 / 16 / 22$ & $01 / 16 / 22$ & $\begin{array}{c}410,599 \\
(399,776-421528)\end{array}$ & $\begin{array}{c}1,913,262 \\
(1891033- \\
1934980)\end{array}$ \\
\hline $\begin{array}{l}\text { South } \\
\text { Sudan }\end{array}$ & 1.51 & 1.54 & $1.19-2.03$ & $01 / 16 / 22$ & $01 / 16 / 22$ & $\begin{array}{c}386,020 \\
(376,478-396244)\end{array}$ & $\begin{array}{c}751,872 \\
(738686- \\
765302)\end{array}$ \\
\hline Tanzania & 2.46 & 2.57 & $1.45-4.31$ & $01 / 15 / 22$ & $01 / 16 / 22$ & $\begin{array}{c}107,265 \\
(95,757-119982)\end{array}$ & $\begin{array}{c}70,197 \\
(60262- \\
80013)\end{array}$ \\
\hline Uganda & 2.30 & 2.34 & $1.67-3.33$ & $01 / 16 / 22$ & $01 / 16 / 22$ & $\begin{array}{c}3,145,602 \\
(3,089,070-3205017)\end{array}$ & $\begin{array}{c}2,425,643 \\
(2375840- \\
2477153)\end{array}$ \\
\hline
\end{tabular}
brackets $(\alpha=0.05)$. 
Time-varying changes caused by government interventions

The COVID-19 pandemic has progressed across Eastern Africa with varying impacts. Hyperparameters introduced into the model allowed for inference of the impact of government interventions at specific time points to control the pandemic. Based on the time varying exponential and stepwise eSIR model, Figure 2 and Figure 3 respectively, indicate an epidemiological trend of COVID-19 under existing preventions in Kenya for the year 2020/2021 and 2021/2022. The exponential model simulated gradual community awareness of interventions by regional governments while the stepwise model simulated NPIs such as school closure, lockdowns and suspension of social gatherings. Additional exponential model projection results are available as supporting information in Figure S1 - S6, while the stepwise model outputs are shown in Figure S7 - S12, for Burundi, Ethiopia, Rwanda, South Sudan, Tanzania, and Uganda respectively.

\section{Figure 2. The exponential model of COVID-19 trend under existing interventions in Kenya. The} black dots left of the blue vertical line denote the observed proportions of the infected and removed compartments. The blue vertical line denotes time $t(0)$. The green and purple vertical lines denote the first and second turning points, respectively. The cyan and salmon colour area denotes the $95 \% \mathrm{CI}$ of the predicted proportions of the infected and removed cases before and after $t(0)$, respectively. The gray and red curves are the posterior mean and median curves. (A, B) Prediction of the infection and removed (recovered and dead) proportions of COVID-19 in 2020/2021 time period; (C, D) Prediction of the infection and removed proportions of COVID-19 in 2021/2022 time period.

Figure 3. The stepwise model of COVID-19 trend under existing interventions Kenya. The black dots left of the blue vertical line denote the observed proportions of the infected and removed compartments. The blue vertical line denotes time $t(0)$. The green and purple vertical lines denote the 
first and second turning points, respectively. The cyan and salmon colour area denotes the $95 \% \mathrm{CI}$ of the predicted proportions of the infected and removed cases before and after $t(0)$, respectively. The gray

Under the basic SIR model without intervention, we observed rampant prevalence of infection and the

Figure 4. The standard state-space SIR model without interventions in Kenya. (A) Prediction of the infection of COVID-19 for 2020/2021 time period; (B) Prediction of the removed compartment for 2020/2021; (C) Plot of the first-order derivatives of the posterior prevalence of infection in 2020/2021. The black curve is the posterior mean of the derivative, and the vertical lines mark times of turning points corresponding respectively to those shown (A, B) and (D, E). The colored semi-transparent rectangles represent the 95\% CI of these turning points. (D) Prediction of the infection of COVID-19 during 2021/2022 
:84 window; (E) Prediction of the removed compartment during 2021/2022 window; (F) Plot of the first-order

:85 derivatives of the posterior prevalence of infection for 2021/2022 time period.

:87 Figure 5. SIR model projection in Kenya with time-varying quarantine. (A) Prediction of COVID-19 :88 infection during 2020/2021 window; (B) Prediction of the removed compartment during 2020/2021 :89 window; (C) Plot of the first-order derivatives of the posterior prevalence of infection in 2020/2021. The window; (F) Plot of the first-order derivatives of the posterior prevalence of infection.

Epidemiological trends with a subset of the population having Covid-19 antibodies and the impact of vaccination campaigns

Herd immunity was simulated using SIR model with time-varying proportion (20\%) of the population assumed to have neutralizing antibodies against COVID-19. We observed a decline in $\mathrm{R}_{0}$ under the assumption that $20 \%$ of the population in EACs had achieved herd immunity by $2021 / 2022$. Furthermore, we also simulated the impact of vaccination on the dynamics of COVID-19. For example, $\mathrm{R}_{0}$ declined from 8.52 to 2.14 under the assumption that $2 \%$ of the Kenyan population is vaccinated (Figure 6). While vaccination eventually contributes to the achievement of herd immunity, vaccination had a bigger impact than herd immunity in lowering $\mathrm{R}_{0}$ for the number of cases and deaths (Figure S25 - S30).

Figure 6. The extended SIR model simulating herd immunity and vaccination campaign in Kenya. (A) Prediction of COVID-19 infection under the assumption that $20 \%$ of the population has antibodies against SARS-COV-2; (B) Prediction of the removed compartment under herd immunity; (C) Projection of the infection in $2021 / 2022$ assuming that $20 \%$ of the population has antibodies against SARS-COV-2; (D) Prediction of COVID-19 infection under the assumption that $2 \%$ of the population is vaccinated; (E) 
i09 Prediction of the removed compartment assuming that $2 \%$ of the population is vaccinated; (F) Projection of

:10 infection in 2021/2022 assuming that $2 \%$ of the population is vaccinated.

i1 1

$: 12$

i13 Validation of the model

¡14 A reliable model results in predicted values being close to the observed data values, which implies a good

Table 3. Validation of the model for prediction of infected COVID-19 cases.

\begin{tabular}{l|l|r|r}
\hline & Mean R0 (95\% CI) & \multicolumn{1}{l}{ RMSE } & \multicolumn{1}{l}{ MAE } \\
\hline Ethiopia & $4.56(2.90-6.45)$ & 9.97 & 10.52 \\
\hline Kenya & $4.02(2.69-5.62)$ & 9.86 & 2.51 \\
\hline Rwanda & $3.62(2.22-5.40)$ & 1.67 & 1.24 \\
\hline Uganda & $4.42(2.47-7.13)$ & 1.98 & 2.53 \\
\hline
\end{tabular}

Figure 7. Validation of the model for prediction of infected COVID-19 cases using the Root Mean square error and Mean Absolute Error. The RMSE and MAE values are indicated in the respective figure subtitles. (A) Uganda; (B) Kenya; (C) Rwanda; (D) Ethiopia.

\section{Discussion}

Following the first reported case of COVID-19 on February 2020 in Egypt, the number of cases gradually increased across African countries causing human and economic damages. Despite the high infectivity of 
the virus, fatalities have remained low particularly during the initial phases of the pandemic. Several explanations to this observation have been proposed including experience with previous pandemics (Ebola virus disease, human immunodeficiency virus, polio, and tuberculosis), demographic factors, host genetics factors, climate and environmental factors [4]. However, to combat the pandemic, many countries had to implement Non-pharmaceutical interventions (NPIs) such as wearing masks, lockdown of cities, stop transports, school closure, social distancing, and hand washing [11].

In this work, we used the extended Susceptible-Exposed-Removed (eSIR) compartmental model [22,23] to project epidemiological trends of COVID-19 infections and the impact of NPIs in seven EACs. Publicly available data from JHU as at $30^{\text {th }}$ July 2021 were used to estimate the transmission rate of the epidemic and to present the trend of infections and fatalities following governmental interventions. The basic reproduction number $\left(\mathrm{R}_{0}\right)$ is an important parameter in epidemic models. For evidence based public health policy formulation, estimation of parameters such as the $\mathrm{R}_{0}$ are of great importance for policy makers to adopt the most efficient and effective interventions in order to contain the pandemic and minimize human and economic damages [14].

The epidemic trend of COVID-19 differs among EACs with infections remaining high while fatalities are low [30]. The $\mathrm{R}_{0}$ posterior values and endpoints in EACs for 2020/2021 and 2021/2022 window provided a snapshot of the trajectories of the disease. $\mathrm{R}_{0}$ is a measure of the contagiousness of COVID-19 and it refers to the number of new infections generated by each infected person [16]. If $\mathrm{R}_{0}<1$, the disease will decline in the population, and if $\mathrm{R}_{0}>1$, the disease will spread faster [17]. However, foreseen risks include underestimation of the disease extend due to asymptomatic cases. We found that interventions during the initial stages of the pandemic had a strong impact on reducing the transmission of the disease with $\mathrm{R}_{0}$ stabilizing with a mean of 2.81 indicating that interventions to lower the transmission fully. For example, after calibrating the model using time-series data of COVID-19 from March 02/2020 to May 01/2020, our predictions revealed a modest $\mathrm{R}_{0}$ value of 2.71 (95\% CI:1.48 - 4.58), 2.75 (95\% CI:1.57 - 4.65), 2.70 (95\% CI:1.54 - 4.67), 3.10 (95\% CI:3.10 - 5.22), 2.71 (95\% CI:2.71 - 4.59), 2.82 (95\% CI:2.82 - 4.90), 2.87 (95\% 
CI:2.87 - 4.79) for Burundi, Ethiopia, Kenya, Rwanda, South Sudan, Tanzania, and Uganda respectively.

However, $\mathrm{R}_{0}$ marginally decreased under the same time period in 2021/2022 projections, except in Burundi and Kenya where the value increased to a mean of 2.84 and 8.52 respectively. Previous studies for COVID19 reported the $\mathrm{R}_{0}$ value between 1.78 (95\% CI: $\left.1.44-2.14\right)$ to 3.46 (95\% CI: $2.81-4.17$ ) in Kenya [31-33].

Indeed, this is within the range of the prediction of the current study, $\mathrm{R}_{0}$ of 2.70 (95\% CI: $\left.1.54-4.67\right)$.

The exponential model which was implemented to reflect increased community-level awareness of NPIs, had more impact in lowering the transmission rate of the disease than the stepwise model that was implemented to reflect governmental interventions at specific timepoints. As the pandemic evolves, the public perceptions and attitudes towards the interventions change [11]. This can be attributed to the timing of introduction of the interventions and social factors that determine adherence to public policies. Moreover, the time point of implementation of NPIs is key to their success in reducing the peak of the epidemic [10]. Overall, the 2021/2022 epidemic trajectories of the seven EACs indicate that they are facing challenges in their efforts to contain community transmission of COVID-19. The projected mean $\mathrm{R}_{0}$ value remains above

$\underline{2}\left(\mathrm{R}_{0}>2\right)$ with the exception of Ethiopia, Rwanda and South Sudan. This is further compounded by the weak health systems, inadequate preparedness and capacity to respond to emerging epidemics. Based on these results, we suggest stringent implementation of intervention policies, such as enforcement of lockdowns, face-mask wearing, long-term surveillance and COVID-19 vaccine roll-out to contain the pandemic. However, we recommend careful interpretation of the $\mathrm{R}_{0}$ values because of the unforeseen risks such as under-estimation of the disease extend due to asymptomatic cases and low testing rate which is not randomized.

Under the current intervention measures, the long-term projection of the extended SIR exponential model indicates that about $0.97 \%, 6.15 \%, 33.94 \%, 3.17 \%, 3.45 \%, 0.18 \%, 6.88 \%$ of the population will be infected by $16^{\text {th }}$ January 2022 in Burundi, Ethiopia, Kenya, Rwanda, South Sudan, Tanzania, and Uganda respectively (Table 2). The high number of recorded cases of COVID-19 could be attributed to the weak health infrastructure, crowded social life and poor personal hygiene. Moreover, disease comorbidities like hypertension, obesity, type II diabetes, HIV, tuberculosis and malaria are highly prevalent in Africa and 
may contribute to the weak immune response to COVID-19 [4,34,35]. The comorbid individuals must be prioritized in terms of healthcare and vaccine roll-out.

Previous predictive models suggested that Africa could be the next hotspot of COVID-19, yet to-date, recorded cases and deaths have remained low. Multiple factors have been attributed to the low COVID-19 reported cases and fatalities in Africa including herd immunity due to antibodies against SARS-COV-2, climate, comorbidities, parasite exposure, and young population structure [30,36,37]. Currently, the reported seroprevalence of antibodies against SARS-CoV-2 range from $0.4 \%$ in Cape Verde and $49 \%$ in antenatal care clinics in Kenya [38,39]. However, most of these factors have not been studied conclusively to establish the interactions of COVID-19 with TB, HIV, malaria and other comorbidities in Africa [4].

Several hypotheses have been proposed to explain the low mortality rates associated with COVID-19 in Africa. For example, the "trained immunity" hypothesis suggests that the Bacillus Calmette-Guérin (BCG) vaccine that has been extensively administered in Africa for tuberculosis confers protection against COVID19 [30]. Genetically, Africans have mutations in the SARS-CoV-2 receptor, angiotensin-converting enzyme-2 (ACE-2) gene, which confers low response to ACE inhibitors and therefore linked to low prevalence of COVID-19 [40]. Furthermore, previous exposure to Plasmodium facliparum and other pathogens is associated with protective immunity and has been linked to a lower prevalence of COVID-19 in malaria-endemic areas. the SARS-CoV-2 virus shares [36,41]. Demographic structure of Africa's population that has a predominantly young population aged below 35 years, and with few comorbidities has been linked to low prevalence to COVID-19. However, such population can be super spreaders of the virus because they are largely asymptomatic [42].

We estimated the herd effect due to genetic factors and COVID-19 vaccination programmes by incorporating proportions of the population with antibodies against SARS-CoV-2 and vaccineimmunization into the simulation of infection dynamics. By assuming that about $20 \%$ of the population in each country had neutralizing antibodies against COVID-19, we observed a significant decline in $\mathrm{R}_{0}$ from 8.52 to 2.62 by January $16^{\text {th }} 2021 / 2022$ in all the EACs. Similarly, $\mathrm{R}_{0}$ declined from 8.52 to 2.14 under the 
assumption that $2 \%$ of the Kenyan population is vaccinated. While vaccination eventually contributes to the

H1 achievement of herd immunity, vaccination had a bigger impact than herd immunity in lowering $\mathrm{R}_{0}$ and

12 hence the number of cases and deaths.

.14 During the initial phases of pandemic, the entire African population had no immunity against COVID-19, H15 hence the virus spread quickly across communities. However, as the disease evolved, gradual immunity H16 developed aided by genetic factors, previous parasite exposure, and a young population structure with few H17 underlying comorbidities. The COVID-19 vaccine has been rolled-out in Africa with 49 countries having H18 administered at least one dose. However, the vaccination coverage required to establish herd immunity of the population having received at least one dose of the vaccine in Burundi, Ethiopia, Kenya, Rwanda, South Sudan, Tanzania, and Uganda respectively as of $12^{\text {th }}$ August $2021[43,44]$. Flattening the curve will require a significant percentage of population to be immunized. In particular, we recommend that countries with high basic reproduction number $\left(\mathrm{R}_{0}>1\right)$ such as Kenya (8.52), Burundi (2.84), Uganda (2.34) and Tanzania (2.57) should increase vaccine coverage required to establish herd immunity against COVID-19 and strictly enforce interventions. However, the current situation is further complicated by weak health systems in EACs, the inequitable vaccine distribution, vaccine hesitancy and negative perception of government interventions. Furthermore, the emergence of COVID-19 variants, such as B.1.617 ("Delta") variant, has led to upsurge of cases due to declining protective immunity or the circulation of immune escape viral variants $[4,45-47]$.

Epidemiological models for projecting infectious disease spread have been used to inform public health policy [20,48-50]. To evaluate the reliability and usefulness of our model, we compared model predictions of the case-counts against the observed data for COVID-19 in Ethiopia, Kenya, Rwanda and Uganda using the Root Mean square error (RMSE) and Mean Absolute Error (MAE). The metrics provided good support to the model fit to the observed COVID-19 cases with larger values indicative of a wider difference between 
the predicted and observed values, hence poor model fit. The modelling techniques that we used in this

137 study to characterize the epidemic dynamics has been successfully applied to the data in India and Wuhan, 138 China, separately $[22,23,29]$. A reliable model results in predicted values close to the observed data values $139[51,52]$.

140 The original eSIR epidemiology model does not provide validation of the predictions [22]. One of the novel 141 contributions to the model was to validate the predictions made by the model using subsequent data from 142 Ethiopia, Kenya, Rwanda and Uganda. A second additional strength was the incorporation of a vaccination 143 compartment into the model to account for vaccine-induced immunity over time. However, we acknowledge 144 that some aspects of these analyses have limitations. For example, the model did not account for under 145 estimation of the reported cases, asymptomatic cases, the population structure, health systems, climate and 146 environmental factors that can affect predictions and forecasts $[5,15,53,54]$.

\section{Conclusion}

\section{Availability of data and materials}


t60 The data of cumulative number of COVID-19 infected cases are available from COVID-19 Data Repository

161 by the Johns Hopkins University Center for Systems Science and Engineering (JHU CCSE) at 162 https://github.com/CSSEGISandData/COVID-19. The R package for eSIR implementation is publicly t63 available at https://github.com/lilywang1988/eSIR.

\section{List of abbreviations}

I66 COVID-19: Coronavirus disease 2019

167 SARS-CoV-2: Severe Acute Respiratory Syndrome Coronavirus 2

t68 NPIs: non-pharmaceutical intervention(s)

169 EACs: Eastern Africa Countries

170 SIR: Susceptible, Infected, Recovered

171 eSIR: extended Susceptible, Infected, Removed

172 SAIR: Susceptible, Antibody, Infected and Removed

$173 \quad \mathbf{R}_{\mathbf{0}}$ : the basic reproduction number

$174 \quad \mathbf{R}(\mathbf{t})$ : time-varying reproduction number

175 MCMC: Markov chain Monte Carlo

176 RMSE: Root Mean square error

177 MAE: Mean Absolute Error (MAE)

178 JHU: Johns Hopkins University

179 IHME: Institute of Health Metrics, Washington, Seattle

180 ICL: Imperial College London

182 References

183 [1] Fauci AS, Lane HC, Redfield RR. Covid-19 — Navigating the Uncharted. N Engl J Med. 2020 
Mar 26;382(13):1268-9.

185 [2] Wang C, Horby PW, Hayden FG, Gao GF. A novel coronavirus outbreak of global health concern. 186 Vol. 395, The Lancet. Elsevier; 2020. p. 470-3.

187 [3] Africa CDC. Coronavirus Disease 2019 (COVID-19) - Africa CDC [Internet]. Vol. 2019, Africa

[4] Tessema SK, Nkengasong JN. Understanding COVID-19 in Africa. Vol. 21, Nature Reviews

[5] Flaxman S, Mishra S, Gandy A, Unwin HJT, Mellan TA, Coupland H, et al. Estimating the effects of non-pharmaceutical interventions on COVID-19 in Europe. Nature. 2020 Jun 8;1-8.

[6] Mboera LEG, Akipede GO, Banerjee A, Cuevas LE, Czypionka T, Khan M, et al. Mitigating lockdown challenges in response to COVID-19 in Sub-Saharan Africa. Vol. 96, International Journal of Infectious Diseases. Elsevier; 2020. p. 308-10.

[7] Gilbert M, Pullano G, Pinotti F, Valdano E, Poletto C, Boëlle PY, et al. Preparedness and vulnerability of African countries against importations of COVID-19: a modelling study. Lancet. 2020 Mar 14;395(10227):871-7.

8] Hagan JE, Ahinkorah BO, Seidu AA, Ameyaw EK, Schack T. Africa's COVID-19 Situation in Focus and Recent Happenings: A Mini Review. Vol. 8, Frontiers in Public Health. Frontiers; 2020. p. 937.

[9] Wu D, Lu J, Liu Y, Zhang Z, Luo L. Positive effects of COVID-19 control measures on influenza prevention. Vol. 95, International Journal of Infectious Diseases. Int J Infect Dis; 2020. p. 345-6.

[10] Imai N, Gaythorpe KAM, Abbott S, Bhatia S, van Elsland S, Prem K, et al. Adoption and impact of non-pharmaceutical interventions for COVID-19. Wellcome Open Res. 2020;5.

[11] Doogan C, Buntine W, Linger H, Brunt S. Public perceptions and attitudes toward covid-19 nonpharmaceutical interventions across six countries: A topic modeling analysis of twitter data. J Med Internet Res. 2020 Sep 1;22(9).

[12] Chaudhry R, Dranitsaris G, Mubashir T, Bartoszko J, Riazi S. A country level analysis measuring 
the impact of government actions, country preparedness and socioeconomic factors on COVID-19 mortality and related health outcomes. EClinicalMedicine. 2020 Aug 1;25.

[13] Jacobi L, Joshi M, Zhu D. Automated Sensitivity Analysis for Bayesian Inference via Markov Chain Monte Carlo: Applications to Gibbs Sampling. SSRN Electron J. 2018;

[14] Taghizadeh L, Karimi A, Heitzinger C. Uncertainty quantification in epidemiological models for the COVID-19 pandemic. Comput Biol Med. 2020 Oct 1;125:104011.

[15] Murray CJ. Forecasting COVID-19 impact on hospital bed-days, ICU-days, ventilator-days and deaths by US state in the next 4 months. 2020;

[16] Delamater PL, Street EJ, Leslie TF, Yang YT, Jacobsen KH. Complexity of the basic reproduction number (R0). Emerg Infect Dis. 2019 Jan 1;25(1):1-4.

[17] Shetty RM, Achaiah NC, Subbarajasetty SB. R0 and Re of COVID-19: Can We Predict When the Pandemic Outbreak will be Contained? Indian J Crit Care Med. 2020 Dec 16;24(11):1125-7.

[18] Osthus D, Hickmann KS, Caragea PC, Higdon D, Del Valle SY. Forecasting seasonal influenza with a state-space SIR model. Ann Appl Stat. 2017 Mar 1;11(1):202-24.

[19] Baroyan O V., Rvachev LA, Basilevsky U V., Ermakov V V., Frank KD, Rvachev MA, et al. Computer modelling of influenza epidemics for the whole country (USSR). Adv Appl Probab. $1971 ; 3(2): 224-6$.

[20] Coburn BJ, Wagner BG, Blower S. Modeling influenza epidemics and pandemics: Insights into the future of swine flu (H1N1). Vol. 7, BMC Medicine. BioMed Central; 2009. p. 30.

[21] Kermack WO, McKendrick AG. Contributions to the mathematical theory of epidemics-I. Bull Math Biol. 1991 Mar;53(1-2):33-55.

[22] Wang L, Zhou Y, He J, Zhu B, Wang F, Tang L, et al. An epidemiological forecast model and software assessing interventions on the COVID-19 epidemic in China. J Data Sci. 2021 Jan 17;18(3):409-32.

[23] Wangping J, Ke H, Yang S, Wenzhe C, Shengshu W, Shanshan Y, et al. Extended SIR Prediction of the Epidemics Trend of COVID-19 in Italy and Compared With Hunan, China. Front Med. 2020 
[24] Mbuvha R, Marwala T. Bayesian inference of COVID-19 spreading rates in South Africa. PLoS One. 2020 Aug 1;15(8):e0237126.

May 6;7:169.

25] Butcher JC. Runge-Kutta Methods. In: Numerical Methods for Ordinary Differential Equations. John Wiley \& Sons, Ltd; 2008. p. 137-316.

[26] Yu X, Dai Q. The Runge-Kutta DG finite element method and the KFVS scheme for compressible flow simulations. Numer Methods Partial Differ Equ. 2006 Nov;22(6):1455-78.

[27] Gholamy A, Kreinovich V, Kosheleva O. Why 70/30 or 80/20 Relation Between Training and Testing Sets : A Pedagogical Explanation. Dep Tech Reports. 2018;1-6.

[28] Plummer M, Stukalov A DM. Bayesian Graphical Models using MCMC - package "rjags." Comprehensive R Archive Network (CRAN); 2019 Nov.

[29] Purkayastha S, Bhattacharyya R, Bhaduri R, Kundu R, Gu X, Salvatore M, et al. A comparison of five epidemiological models for transmission of SARS-CoV-2 in India. Vol. 21, BMC Infectious Diseases. BMC Infectious Diseases; 2021. p. 1-23.

[30] Ghosh D, Jonathan A, Mersha TB. COVID-19 Pandemic: The African Paradox. J Glob Health. 2020;10(2):1-6.

[31] Quaife M, Van Zandvoort K, Gimma A, Shah K, McCreesh N, Prem K, et al. The impact of COVID-19 control measures on social contacts and transmission in Kenyan informal settlements. BMC Med. 2020;18(1).

[32] Brand SPC, Aziza R, Kombe IK, Agoti CN, Hilton J, Rock KS, et al. Forecasting the scale of the COVID-19 epidemic in Kenya. medRxiv. 2020 Apr 16;2020.04.09.20059865.

[33] Mwalili S, Kimathi M, Ojiambo V, Gathungu D, Achia T. Age-structured Impact of Mitigation Strategies on COVID-19 Severity and Deaths in Kenya. ResearchSquare. 2020;1-14.

[34] Das S, K.R. A, Birangal SR, Nikam AN, Pandey A, Mutalik S, et al. Role of comorbidities like diabetes on severe acute respiratory syndrome coronavirus-2: A review. Vol. 258, Life Sciences. Elsevier Inc.; 2020. 


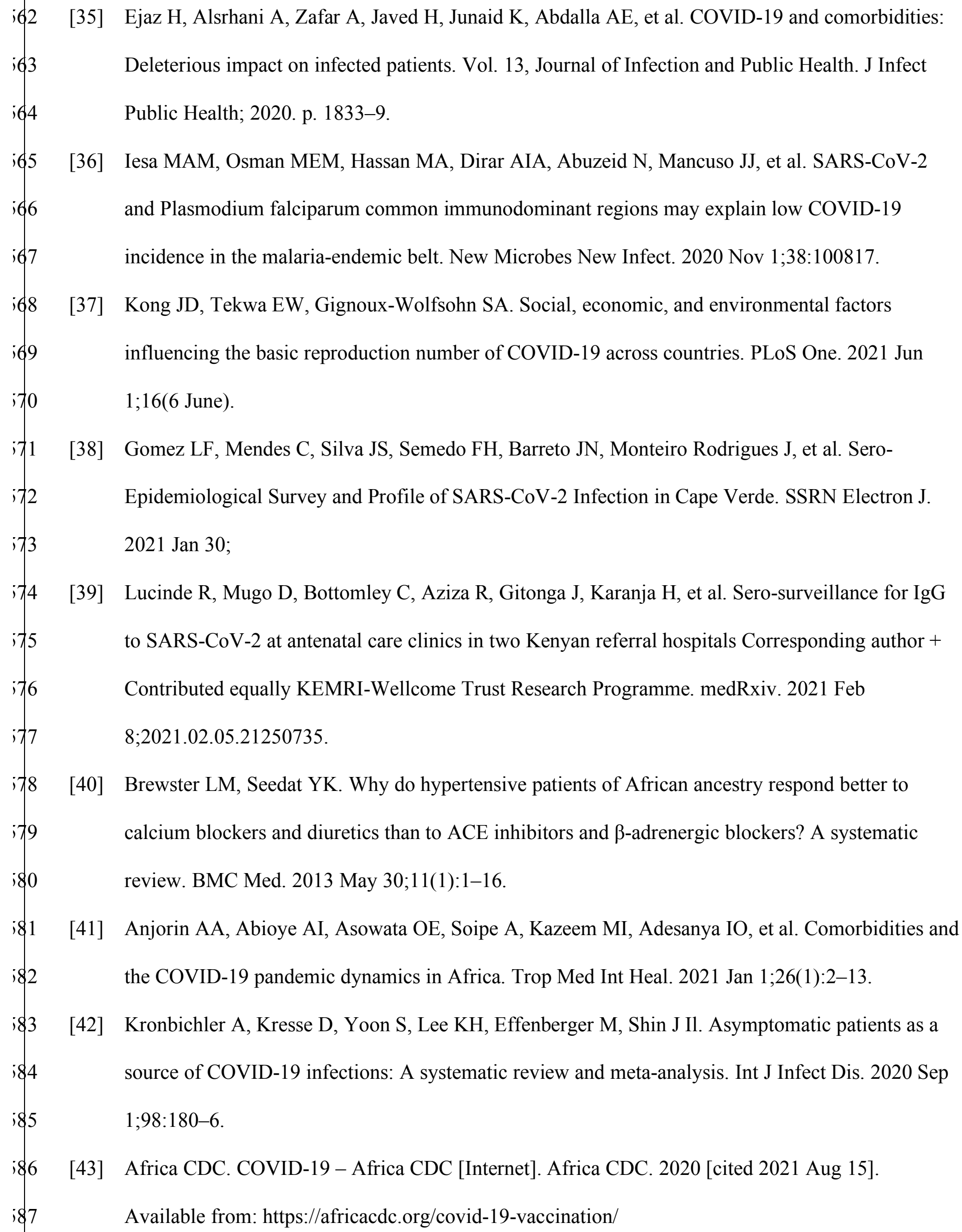

[35] Ejaz H, Alsrhani A, Zafar A, Javed H, Junaid K, Abdalla AE, et al. COVID-19 and comorbidities: Deleterious impact on infected patients. Vol. 13, Journal of Infection and Public Health. J Infect Public Health; 2020. p. 1833-9.

[36] Iesa MAM, Osman MEM, Hassan MA, Dirar AIA, Abuzeid N, Mancuso JJ, et al. SARS-CoV-2 and Plasmodium falciparum common immunodominant regions may explain low COVID-19 incidence in the malaria-endemic belt. New Microbes New Infect. 2020 Nov 1;38:100817.

[37] Kong JD, Tekwa EW, Gignoux-Wolfsohn SA. Social, economic, and environmental factors influencing the basic reproduction number of COVID-19 across countries. PLoS One. 2021 Jun $1 ; 16(6$ June).

[38] Gomez LF, Mendes C, Silva JS, Semedo FH, Barreto JN, Monteiro Rodrigues J, et al. SeroEpidemiological Survey and Profile of SARS-CoV-2 Infection in Cape Verde. SSRN Electron J. 2021 Jan 30;

[39] Lucinde R, Mugo D, Bottomley C, Aziza R, Gitonga J, Karanja H, et al. Sero-surveillance for IgG

[40] Brewster LM, Seedat YK. Why do hypertensive patients of African ancestry respond better to calcium blockers and diuretics than to ACE inhibitors and $\beta$-adrenergic blockers? A systematic review. BMC Med. 2013 May 30;11(1):1-16.

[41] Anjorin AA, Abioye AI, Asowata OE, Soipe A, Kazeem MI, Adesanya IO, et al. Comorbidities and the COVID-19 pandemic dynamics in Africa. Trop Med Int Heal. 2021 Jan 1;26(1):2-13.

[42] Kronbichler A, Kresse D, Yoon S, Lee KH, Effenberger M, Shin J Il. Asymptomatic patients as a source of COVID-19 infections: A systematic review and meta-analysis. Int J Infect Dis. 2020 Sep $1 ; 98: 180-6$.

[43] Africa CDC. COVID-19 - Africa CDC [Internet]. Africa CDC. 2020 [cited 2021 Aug 15]. Available from: https://africacdc.org/covid-19-vaccination/ 


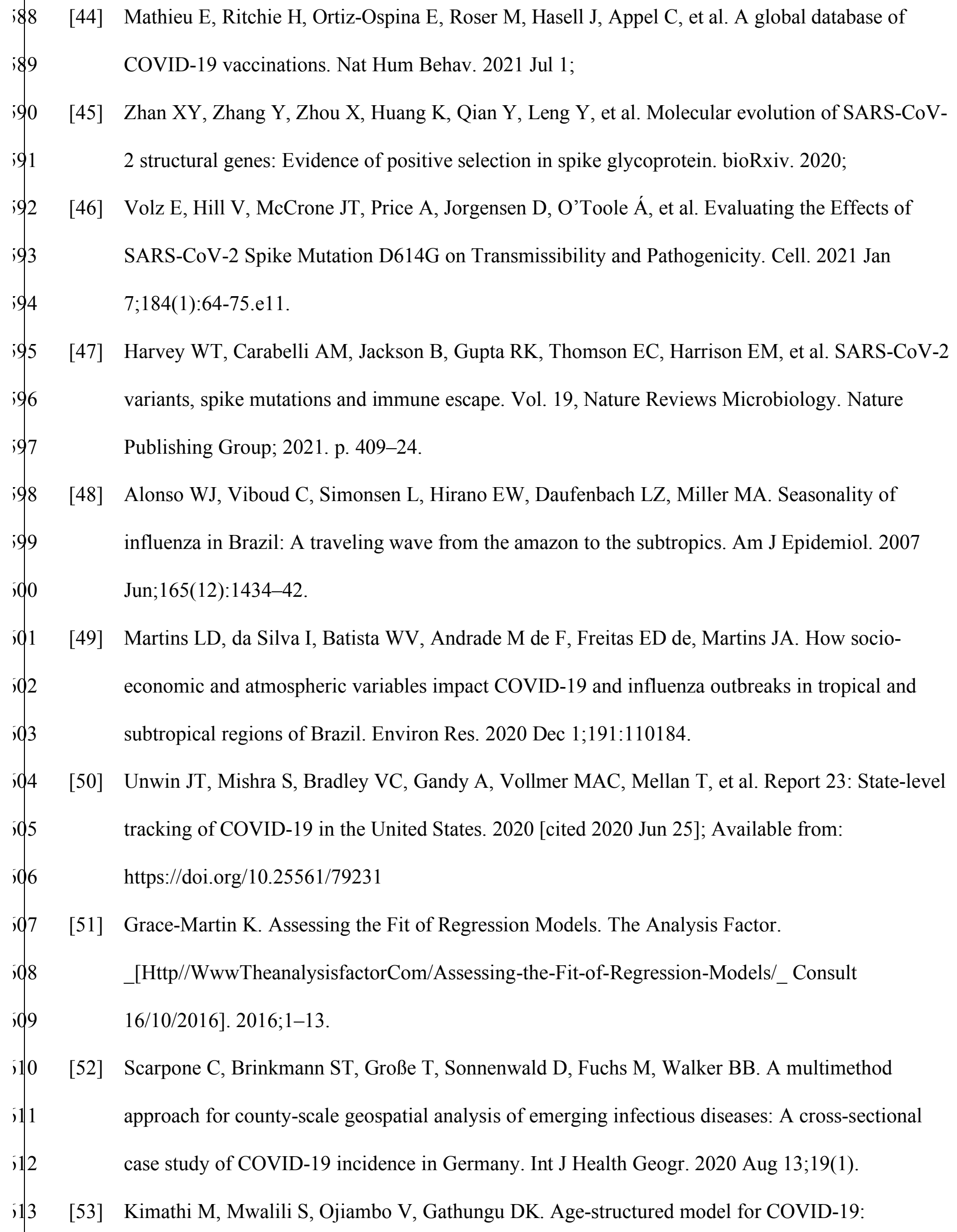

[44] Mathieu E, Ritchie H, Ortiz-Ospina E, Roser M, Hasell J, Appel C, et al. A global database of COVID-19 vaccinations. Nat Hum Behav. 2021 Jul 1;

[46] Volz E, Hill V, McCrone JT, Price A, Jorgensen D, O’Toole Á, et al. Evaluating the Effects of 2 structural genes: Evidence of positive selection in spike glycoprotein. bioRxiv. 2020;

[45] Zhan XY, Zhang Y, Zhou X, Huang K, Qian Y, Leng Y, et al. Molecular evolution of SARS-CoVSARS-CoV-2 Spike Mutation D614G on Transmissibility and Pathogenicity. Cell. 2021 Jan 7;184(1):64-75.e11.

[47] Harvey WT, Carabelli AM, Jackson B, Gupta RK, Thomson EC, Harrison EM, et al. SARS-CoV-2 variants, spike mutations and immune escape. Vol. 19, Nature Reviews Microbiology. Nature Publishing Group; 2021. p. 409-24.

[48] Alonso WJ, Viboud C, Simonsen L, Hirano EW, Daufenbach LZ, Miller MA. Seasonality of influenza in Brazil: A traveling wave from the amazon to the subtropics. Am J Epidemiol. 2007 Jun;165(12):1434-42.

[49] Martins LD, da Silva I, Batista WV, Andrade M de F, Freitas ED de, Martins JA. How socioeconomic and atmospheric variables impact COVID-19 and influenza outbreaks in tropical and subtropical regions of Brazil. Environ Res. 2020 Dec 1;191:110184.

[50] Unwin JT, Mishra S, Bradley VC, Gandy A, Vollmer MAC, Mellan T, et al. Report 23: State-level tracking of COVID-19 in the United States. 2020 [cited 2020 Jun 25]; Available from: https://doi.org/10.25561/79231

[51] Grace-Martin K. Assessing the Fit of Regression Models. The Analysis Factor. _[Http//WwwTheanalysisfactorCom/Assessing-the-Fit-of-Regression-Models/_Consult 16/10/2016]. 2016;1-13.

[52] Scarpone C, Brinkmann ST, Große T, Sonnenwald D, Fuchs M, Walker BB. A multimethod approach for county-scale geospatial analysis of emerging infectious diseases: A cross-sectional case study of COVID-19 incidence in Germany. Int J Health Geogr. 2020 Aug 13;19(1).

53] Kimathi M, Mwalili S, Ojiambo V, Gathungu DK. Age-structured model for COVID-19: 
i14 Effectiveness of social distancing and contact reduction in Kenya. Infect Dis Model. 2021 Jan

i15 $1 ; 6: 15-23$.

il [54] Mwalili S, Kimathi M, Ojiambo V, Gathungu D, Mbogo R. SEIR model for COVID-19 dynamics incorporating the environment and social distancing. BMC Res Notes. $2020 \mathrm{Jul}$ 23;13(1).

¡19 Acknowledgements

i20 Not applicable.

\section{i22 Funding}

i23 This work received financial support from the following organizations and agencies: BioInnovate Africa, i24 International Centre of Insect Physiology and Ecology (icipe), grant number: B8401F; The European Union; i25 the Swedish International Development Cooperation Agency (Sida); the Swiss Agency for Development i26 and Cooperation (SDC); the Federal Democratic Republic of Ethiopia; and the Government of the Republic i27 of Kenya. The views expressed herein do not necessarily reflect the official opinion of the donors.

\section{i29 Author information}

Mark Wamalwa and Henri E.Z. Tonnang contributed equally to this work.

\section{Affiliations}

${ }^{1}$ International Centre of Insect Physiology and Ecology (icipe), P.O. Box 30772-00100, Nairobi, Kenya.

${ }^{2}$ Department of Biochemistry, Microbiology and Biotechnology, Kenyatta University, Kenya.

\section{Contributions}


H.E.Z. Tonnang conceptualized and designed the work. M.W. acquired, analyzed and interpreted the data.

i38 Both authors contributed equally in drafting, reviewing of the article and final approval of the version to i39 be published.

\section{¡41 Ethics declarations}

\section{$i 42$ Ethics approval and consent to participate}

i43 This is an epidemiological modelling research and therefore presents secondary data. No ethical approvals i44 were required.

\section{¡45 Consent for publication}

¡46 Not applicable.

\section{Competing interests}

i48 The authors have declared that no competing interests exist.

\section{Supplementary Information}

S1 Figure. The exponential model of COVID-19 under existing interventions in Burundi. The black dots left of the blue vertical line denote the observed proportions of the infected and removed compartments.

The blue vertical line denotes time $t(0)$. The green and purple vertical lines denote the first and second turning points, respectively. The cyan and salmon colour area denotes the $95 \% \mathrm{CI}$ of the predicted proportions of the infected and removed cases before and after $t(0)$, respectively. The gray and red curves are the posterior mean and median curves. (A, B) Prediction of the infection and removed (recovered and dead) proportions of COVID-19 during 2020/2021 window; (C, D) Prediction of the infection and removed proportions of COVID-19 during 2021/2022 window. 
S2 Figure. The exponential model of COVID-19 under existing interventions in Ethiopia. The black

dots left of the blue vertical line denote the observed proportions of the infected and removed compartments.

The blue vertical line denotes time $t(0)$. The green and purple vertical lines denote the first and second turning points, respectively. The cyan and salmon colour area denotes the $95 \%$ CI of the predicted proportions of the infected and removed cases before and after $t(0)$, respectively. The gray and red curves

\section{S4 Figure. The exponential model of COVID-19 under existing interventions in South Sudan. The} black dots left of the blue vertical line denote the observed proportions of the infected and removed compartments. The blue vertical line denotes time $t(0)$. The green and purple vertical lines denote the first and second turning points, respectively. The cyan and salmon colour area denotes the $95 \%$ CI of the predicted proportions of the infected and removed cases before and after $t(0)$, respectively. The gray and red curves are the posterior mean and median curves. (A, B) Prediction of the infection and removed (recovered and dead) proportions of COVID-19 during 2020/2021 window; (C, D) Prediction of the infection and removed proportions of COVID-19 during 2021/2022 window. 
S5 Figure. The exponential model of COVID-19 under existing interventions in Tanzania. The black

dots left of the blue vertical line denote the observed proportions of the infected and removed compartments.

The blue vertical line denotes time $t(0)$. The green and purple vertical lines denote the first and second

turning points, respectively. The cyan and salmon colour area denotes the $95 \% \mathrm{CI}$ of the predicted

proportions of the infected and removed cases before and after $t(0)$, respectively. The gray and red curves

are the posterior mean and median curves. $(\mathrm{A}, \mathrm{B})$ Prediction of the infection and removed (recovered and dead) proportions of COVID-19 in 2020/2021 window; (C, D) Prediction of the infection and removed proportions of COVID-19 in 2021/2022 window.

S6 Figure. The exponential model of COVID-19 under existing interventions in Uganda. The black dots left of the blue vertical line denote the observed proportions of the infected and removed compartments.

The blue vertical line denotes time $t(0)$. The green and purple vertical lines denote the first and second turning points, respectively. The cyan and salmon colour area denotes the $95 \% \mathrm{CI}$ of the predicted proportions of the infected and removed cases before and after $t(0)$, respectively. The gray and red curves are the posterior mean and median curves. $(\mathrm{A}, \mathrm{B})$ Prediction of the infection and removed (recovered and dead) proportions of COVID-19 in 2020/2021 window; (C, D) Prediction of the infection and removed proportions of COVID-19 in 2021/2022 window.

S7 Figure. The stepwise model of COVID-19 trend under existing interventions Burundi. (A, B) Prediction of the infection and removed (recovered and dead) proportions of COVID-19 during 2020/2021 window; (C, D) Prediction of the infection and removed proportions of COVID-19 during 2021/2022 window.

S8 Figure. The stepwise model of COVID-19 trend under existing interventions Ethiopia. (A, B) Prediction of the infection and removed (recovered and dead) proportions of COVID-19 during 2020/2021 
'12 window; (C, D) Prediction of the infection and removed proportions of COVID-19 during 2021/2022

13 window.

14

'15 S9 Figure. The stepwise model of COVID-19 trend under existing interventions Rwanda. (A, B)

${ }^{\prime} 16$ Prediction of the infection and removed (recovered and dead) proportions of COVID-19 during 2020/2021

'17 window; (C, D) Prediction of the infection and removed proportions of COVID-19 during 2021/2022

${ }^{\prime} 18$ window. window.

S10 Figure. The stepwise model of COVID-19 trend under existing interventions South Sudan. (A, B) Prediction of the infection and removed (recovered and dead) proportions of COVID-19 during 2020/2021 window; (C, D) Prediction of the infection and removed proportions of COVID-19 during 2021/2022

S11 Figure. The stepwise model of COVID-19 trend under existing interventions Tanzania. (A, B) Prediction of the infection and removed (recovered and dead) proportions of COVID-19 during 2020/2021 window; (C, D) Prediction of the infection and removed proportions of COVID-19 during 2021/2022 window.

\section{S12 Figure. The stepwise model of COVID-19 trend under existing interventions Uganda. (A, B)} Prediction of the infection and removed (recovered and dead) proportions of COVID-19 during 2020/2021 window; (C, D) Prediction of the infection and removed proportions of COVID-19 during 2021/2022 window.

S13 Figure. The standard state-space SIR model without interventions in Burundi. (A, B) Prediction of the infection and removed (recovered and dead) proportions of COVID-19 during 2020/2021 window; (C) Plot of the first-order derivatives of the posterior prevalence of infection in 2020/2021. (D, E) Prediction 
of the infection and removed proportions of COVID-19 during 2021/2022 window. (F) Plot of the first-

39 order derivatives of the posterior prevalence of infection for 2021/2022 time period. The colored semi-

$' 40$ transparent rectangles represent the $95 \%$ CI of these turning points. The black curve is the posterior mean

'41 of the derivative, and the vertical lines mark times of turning points corresponding respectively to those

'42 shown (A, B) and (D, E).

S14 Figure. The standard state-space SIR model without interventions in Ethiopia. (A, B) Prediction of the infection and removed (recovered and dead) proportions of COVID-19 during 2020/2021 window; (C) Plot of the first-order derivatives of the posterior prevalence of infection in 2020/2021. (D, E) Prediction of the infection and removed proportions of COVID-19 during 2021/2022 window. (F) Plot of the firstorder derivatives of the posterior prevalence of infection for 2021/2022 time period. The colored semitransparent rectangles represent the $95 \% \mathrm{CI}$ of these turning points. The black curve is the posterior mean of the derivative, and the vertical lines mark times of turning points corresponding respectively to those shown (A, B) and (D, E).

S15 Figure. The standard state-space SIR model without interventions in Rwanda. (A, B) Prediction of the infection and removed (recovered and dead) proportions of COVID-19 during 2020/2021 window; (C) Plot of the first-order derivatives of the posterior prevalence of infection in 2020/2021. (D, E) Prediction of the infection and removed proportions of COVID-19 during 2021/2022 window. (F) Plot of the firstorder derivatives of the posterior prevalence of infection for 2021/2022 time period. The colored semitransparent rectangles represent the $95 \% \mathrm{CI}$ of these turning points. The black curve is the posterior mean of the derivative, and the vertical lines mark times of turning points corresponding respectively to those shown (A, B) and (D, E). 
S16 Figure. The standard state-space SIR model without interventions in South Sudan. (A, B)

'64 Prediction of the infection and removed (recovered and dead) proportions of COVID-19 during 2020/2021

'65 window; (C) Plot of the first-order derivatives of the posterior prevalence of infection in 2020/2021. (D, E)

'66 Prediction of the infection and removed proportions of COVID-19 during 2021/2022 window. (F) Plot of

'67 the first-order derivatives of the posterior prevalence of infection for 2021/2022 time period. The colored

'68 semi-transparent rectangles represent the 95\% CI of these turning points. The black curve is the posterior

'69 mean of the derivative, and the vertical lines mark times of turning points corresponding respectively to

70 those shown $(\mathrm{A}, \mathrm{B})$ and $(\mathrm{D}, \mathrm{E})$. order derivatives of the posterior prevalence of infection for 2021/2022 time period. The colored semi-

S17 Figure. The standard state-space SIR model without interventions in Tanzania. (A, B) Prediction of the infection and removed (recovered and dead) proportions of COVID-19 during 2020/2021 window; (C) Plot of the first-order derivatives of the posterior prevalence of infection in 2020/2021. (D, E) Prediction of the infection and removed proportions of COVID-19 during 2021/2022 window. (F) Plot of the firsttransparent rectangles represent the $95 \% \mathrm{CI}$ of these turning points. The black curve is the posterior mean of the derivative, and the vertical lines mark times of turning points corresponding respectively to those shown $(A, B)$ and $(D, E)$.

\section{S18 Figure. The standard state-space SIR model without interventions in Uganda. (A, B) Prediction} of the infection and removed (recovered and dead) proportions of COVID-19 during 2020/2021 window; (C) Plot of the first-order derivatives of the posterior prevalence of infection in 2020/2021. (D, E) Prediction of the infection and removed proportions of COVID-19 during 2021/2022 window. (F) Plot of the firstorder derivatives of the posterior prevalence of infection for 2021/2022 time period. The colored semitransparent rectangles represent the $95 \% \mathrm{CI}$ of these turning points. The black curve is the posterior mean of the derivative, and the vertical lines mark times of turning points corresponding respectively to those shown (A, B) and (D, E). 
S19 Figure. SIR model projection in Burundi with time-varying quarantine. (A) Prediction of COVID-

19 infection during 2020/2021 window; (B) Prediction of the removed compartment during 2020/2021

window; (C) Plot of the first-order derivatives of the posterior prevalence of infection in 2020/2021. The

colored semi-transparent rectangles represent the $95 \% \mathrm{CI}$ of these turning points. (D) Prediction of the

infection of COVID-19 for 2021/2022; (E) Prediction of the removed compartment during 2021/2022

window; (F) Plot of the first-order derivatives of the posterior prevalence of infection during 2021/2022 window.

S20 Figure. SIR model projection in Ethiopia with time-varying quarantine. (A) Prediction of COVID-

19 infection during 2020/2021 window; (B) Prediction of the removed compartment during 2020/2021 window; (C) Plot of the first-order derivatives of the posterior prevalence of infection in 2020/2021. The colored semi-transparent rectangles represent the 95\% CI of these turning points. (D) Prediction of the infection of COVID-19 for 2021/2022; (E) Prediction of the removed compartment during 2021/2022 window; (F) Plot of the first-order derivatives of the posterior prevalence of infection during 2021/2022 window.

S21 Figure. SIR model projection in Rwanda with time-varying quarantine. (A) Prediction of COVID-

19 infection during 2020/2021 window; (B) Prediction of the removed compartment during 2020/2021 window; (C) Plot of the first-order derivatives of the posterior prevalence of infection in 2020/2021. The colored semi-transparent rectangles represent the $95 \% \mathrm{CI}$ of these turning points. (D) Prediction of the infection of COVID-19 for 2021/2022;

(E) Prediction of the removed compartment during 2021/2022 window; (F) Plot of the first-order derivatives of the posterior prevalence of infection during 2021/2022 ;12 window. 
¡14 S22 Figure. SIR model projection in South Sudan with time-varying quarantine. (A) Prediction of

¿15 COVID-19 infection during 2020/2021 window; (B) Prediction of the removed compartment during ;16 2020/2021 window; (C) Plot of the first-order derivatives of the posterior prevalence of infection in ;17 2020/2021. The colored semi-transparent rectangles represent the 95\% CI of these turning points. (D) ¿18 Prediction of the infection of COVID-19 for 2021/2022; (E) Prediction of the removed compartment during ;19 2021/2022 window; (F) Plot of the first-order derivatives of the posterior prevalence of infection during :20 2021/2022 window.

S24 Figure. SIR model projection in Uganda with time-varying quarantine. (A) Prediction of COVID-

S23 Figure. SIR model projection in Tanzania with time-varying quarantine. (A) Prediction of COVID-19 infection during 2020/2021 window; (B) Prediction of the removed compartment during 2020/2021 window; (C) Plot of the first-order derivatives of the posterior prevalence of infection in 2020/2021. The colored semi-transparent rectangles represent the $95 \%$ CI of these turning points. (D) Prediction of the infection of COVID-19 for 2021/2022; (E) Prediction of the removed compartment during 2021/2022 window; (F) Plot of the first-order derivatives of the posterior prevalence of infection during 2021/2022 window.

19 infection during 2020/2021 window; (B) Prediction of the removed compartment during 2020/2021 window; (C) Plot of the first-order derivatives of the posterior prevalence of infection in 2020/2021. The colored semi-transparent rectangles represent the $95 \% \mathrm{CI}$ of these turning points. (D) Prediction of the infection of COVID-19 for 2021/2022; (E) Prediction of the removed compartment during 2021/2022 window; (F) Plot of the first-order derivatives of the posterior prevalence of infection during 2021/2022 window. 
S25 Figure. The extended SIR model simulating herd immunity and vaccination campaign in

Burundi. (A) Prediction of COVID-19 infection under the assumption that $20 \%$ of the population has

antibodies against SARS-COV-2; (B) Prediction of the removed compartment under herd immunity; (C)

Projection of the infection in $2021 / 2022$ assuming that $20 \%$ of the population has antibodies against SARS-

COV-2; (D) Prediction of COVID-19 infection under the assumption that $2 \%$ of the population is

vaccinated; (E) Prediction of the removed compartment assuming that $2 \%$ of the population is vaccinated;

(F) Projection of infection in $2021 / 2022$ assuming that $2 \%$ of the population is vaccinated.

S26 Figure. The extended SIR model simulating herd immunity and vaccination campaign in Ethiopia. (A) Prediction of COVID-19 infection under the assumption that $20 \%$ of the population has antibodies against SARS-COV-2; (B) Prediction of the removed compartment under herd immunity; (C) Projection of the infection in $2021 / 2022$ assuming that $20 \%$ of the population has antibodies against SARSCOV-2; (D) Prediction of COVID-19 infection under the assumption that $2 \%$ of the population is vaccinated; (E) Prediction of the removed compartment assuming that $2 \%$ of the population is vaccinated; (F) Projection of infection in $2021 / 2022$ assuming that $2 \%$ of the population is vaccinated.

S27 Figure. The extended SIR model simulating herd immunity and vaccination campaign in Rwanda. (A) Prediction of COVID-19 infection under the assumption that $20 \%$ of the population has antibodies against SARS-COV-2; (B) Prediction of the removed compartment under herd immunity; (C) Projection of the infection in $2021 / 2022$ assuming that $20 \%$ of the population has antibodies against SARSCOV-2; (D) Prediction of COVID-19 infection under the assumption that $2 \%$ of the population is vaccinated; (E) Prediction of the removed compartment assuming that $2 \%$ of the population is vaccinated; (F) Projection of infection in $2021 / 2022$ assuming that $2 \%$ of the population is vaccinated.

S28 Figure. The extended SIR model simulating herd immunity and vaccination campaign in South Sudan. (A) Prediction of COVID-19 infection under the assumption that $20 \%$ of the population has 
antibodies against SARS-COV-2; (B) Prediction of the removed compartment under herd immunity; (C)

Projection of the infection in $2021 / 2022$ assuming that $20 \%$ of the population has antibodies against SARS-

COV-2; (D) Prediction of COVID-19 infection under the assumption that $2 \%$ of the population is

vaccinated; (E) Prediction of the removed compartment assuming that $2 \%$ of the population is vaccinated;

(F) Projection of infection in $2021 / 2022$ assuming that $2 \%$ of the population is vaccinated.

\section{S30 Figure. The extended SIR model simulating herd immunity and vaccination campaign in Uganda.}

(A) Prediction of COVID-19 infection under the assumption that $20 \%$ of the population has antibodies against SARS-COV-2; (B) Prediction of the removed compartment under herd immunity; (C) Projection of the infection in $2021 / 2022$ assuming that $20 \%$ of the population has antibodies against SARS-COV-2; (D) Prediction of COVID-19 infection under the assumption that $2 \%$ of the population is vaccinated; (E) Prediction of the removed compartment assuming that $2 \%$ of the population is vaccinated; (F) Projection of infection in $2021 / 2022$ assuming that $2 \%$ of the population is vaccinated. 
Figures

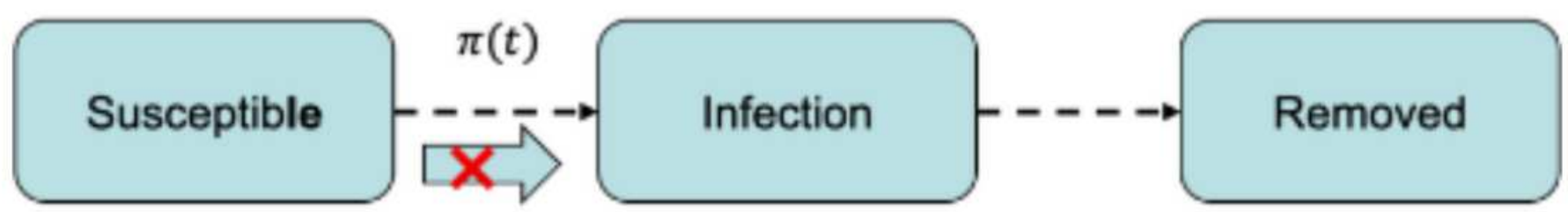

$$
\frac{d \theta_{t}^{S}}{d t}=-\beta \pi(t) \theta_{t}^{S} \theta_{t}^{I}, \frac{d \theta_{t}^{I}}{d t}=-\beta \pi(t) \theta_{t}^{S} \theta_{t}^{I}-\gamma \theta_{t}^{I}, \frac{d \theta_{t}^{R}}{d t}=\gamma \theta_{t}^{I}
$$

\section{Figure 1}

The extended Susceptible-Exposed-Removed (eSIR) basic model. The transmission rate modifier $\pi(t)$ takes on values according to actual interventions in different countries [22]. 

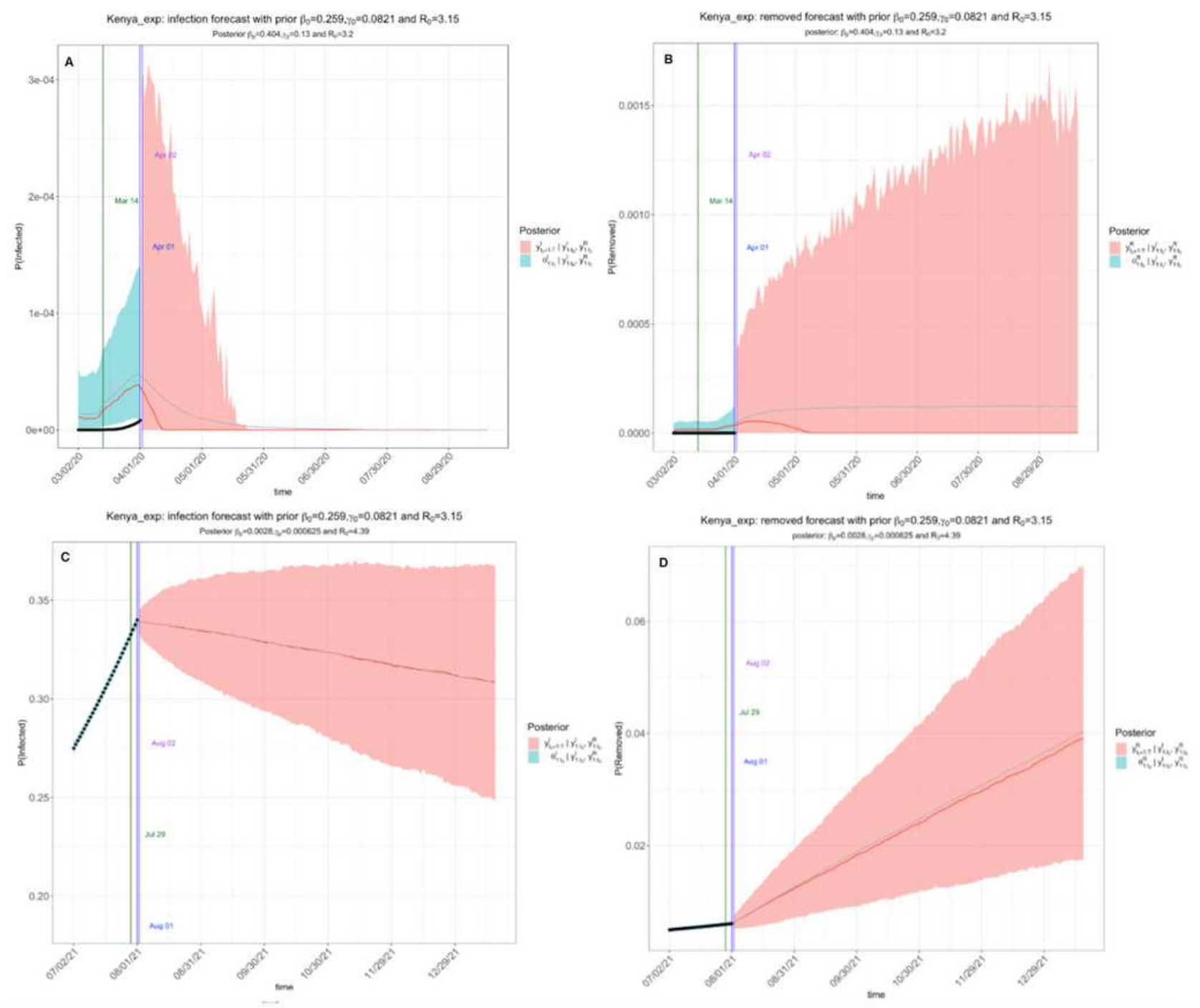

\section{Figure 2}

The exponential model of COVID-19 trend under existing interventions in Kenya. The black dots left of the blue vertical line denote the observed proportions of the infected and removed compartments. The blue vertical line denotes time $t(0)$. The green and purple vertical lines denote the first and second turning points, respectively. The cyan and salmon colour area denotes the $95 \% \mathrm{Cl}$ of the predicted proportions of the infected and removed cases before and after $\mathrm{t}(0)$, respectively. The gray and red curves are the posterior mean and median curves. (A, B) Prediction of the infection and removed (recovered and dead) proportions of COVID-19 in 2020/2021 time period; (C, D) Prediction of the infection and removed proportions of COVID-19 in 2021/2022 time period. 

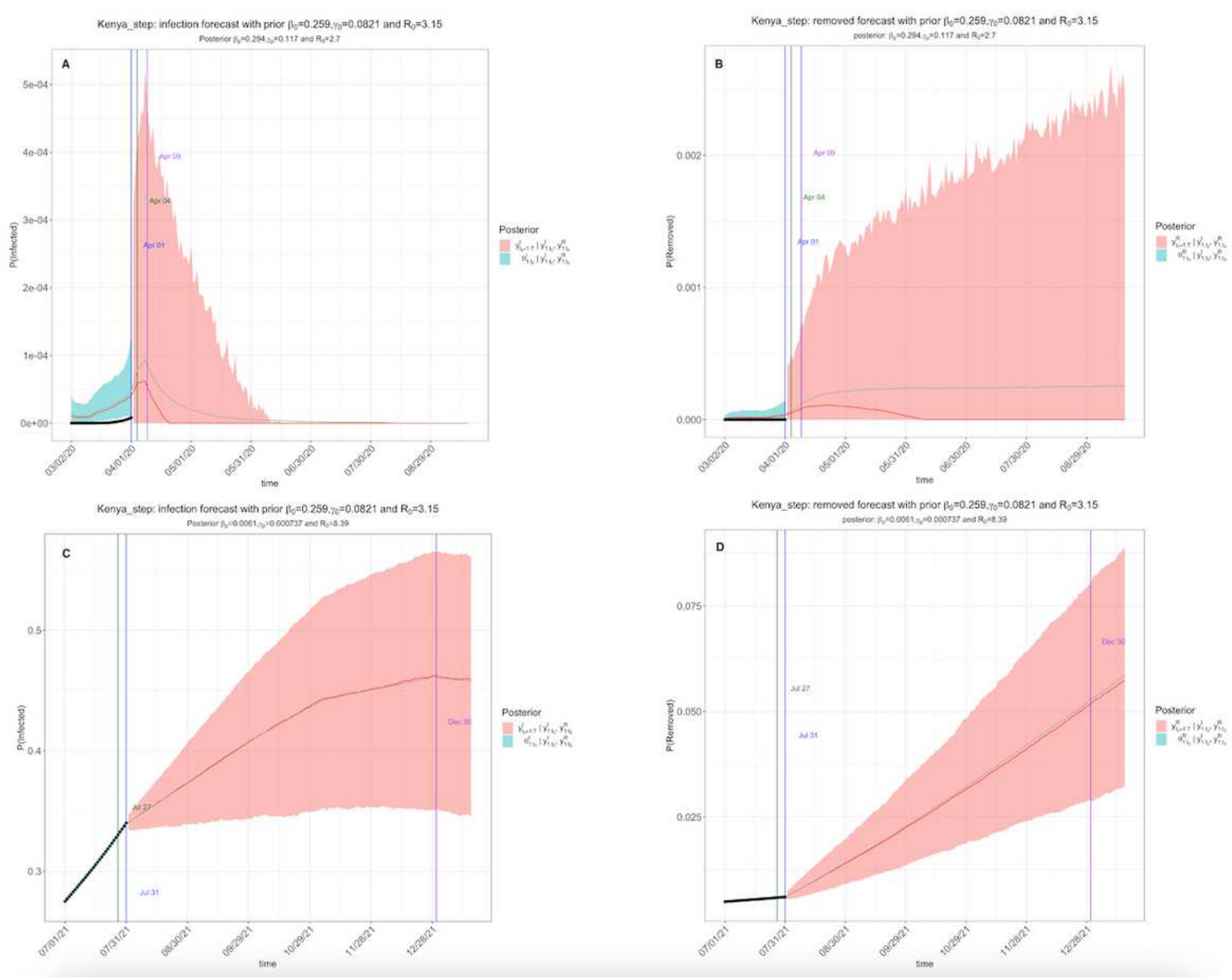

Figure 3

The stepwise model of COVID-19 trend under existing interventions Kenya. The black dots left of the blue vertical line denote the observed proportions of the infected and removed compartments. The blue vertical line denotes time $t(0)$. The green and purple vertical lines denote the first and second turning points, respectively. The cyan and salmon colour area denotes the $95 \% \mathrm{Cl}$ of the predicted proportions of the infected and removed cases before and after $t(0)$, respectively. The gray and red curves are the posterior mean and median curves. (A, B) Prediction of the infection and removed (recovered and dead) proportions of COVID-19 in 2020/2021; (C, D) Prediction of the infection and removed compartments of COVID-19 in 2021/2022. 

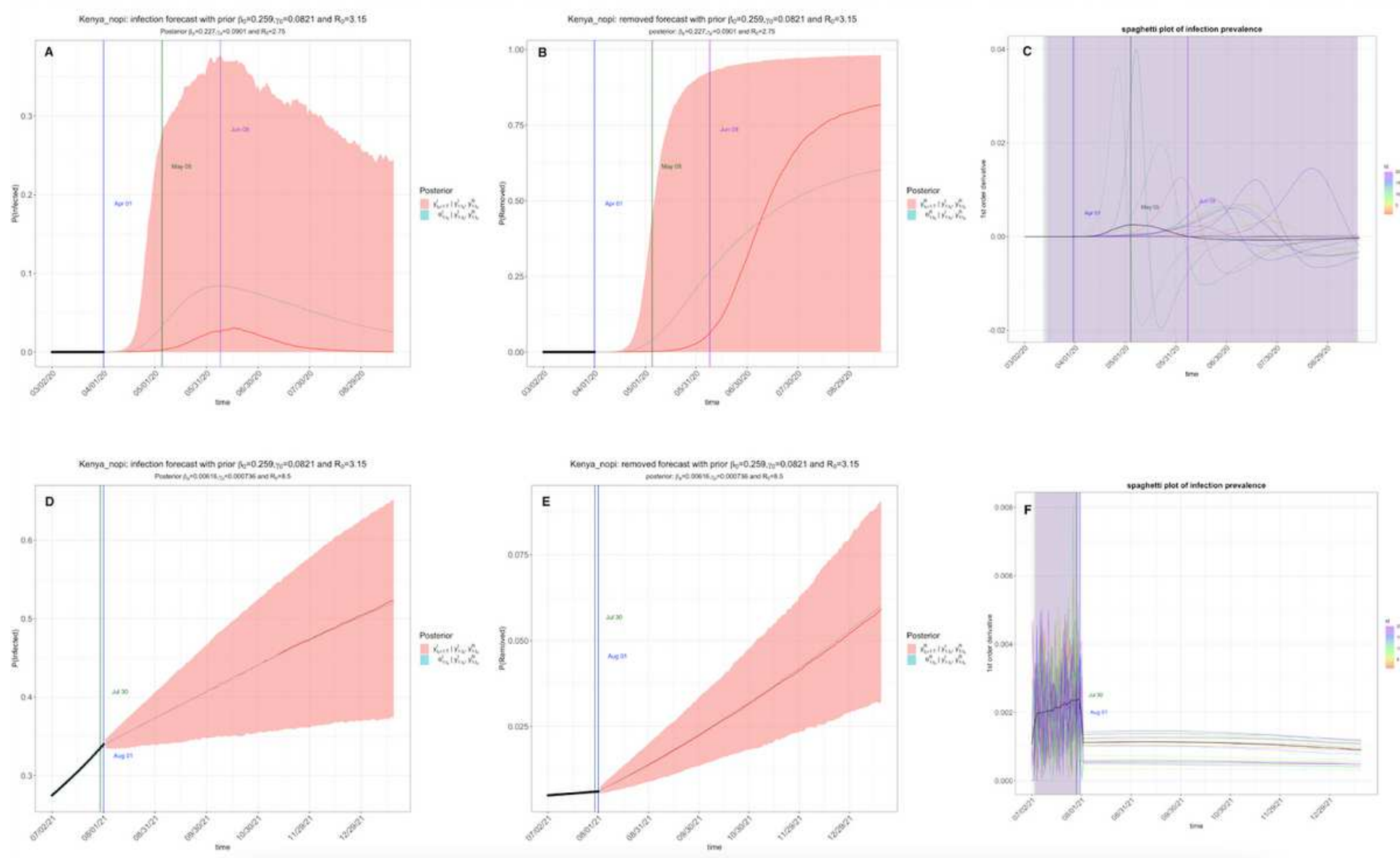

\section{Figure 4}

The standard state-space SIR model without interventions in Kenya. (A) Prediction of the infection of COVID-19 for 2020/2021 time period; (B) Prediction of the removed compartment for 2020/2021; (C) Plot of the first-order derivatives of the posterior prevalence of infection in 2020/2021. The black curve is the posterior mean of the derivative, and the vertical lines mark times of turning points corresponding respectively to those shown $(A, B)$ and $(D, E)$. The colored semi-transparent rectangles represent the $95 \%$ $\mathrm{Cl}$ of these turning points. (D) Prediction of the infection of COVID-19 during 2021/2022 window; (E) Prediction of the removed compartment during 2021/2022 window; (F) Plot of the first-order derivatives of the posterior prevalence of infection for 2021/2022 time period. 


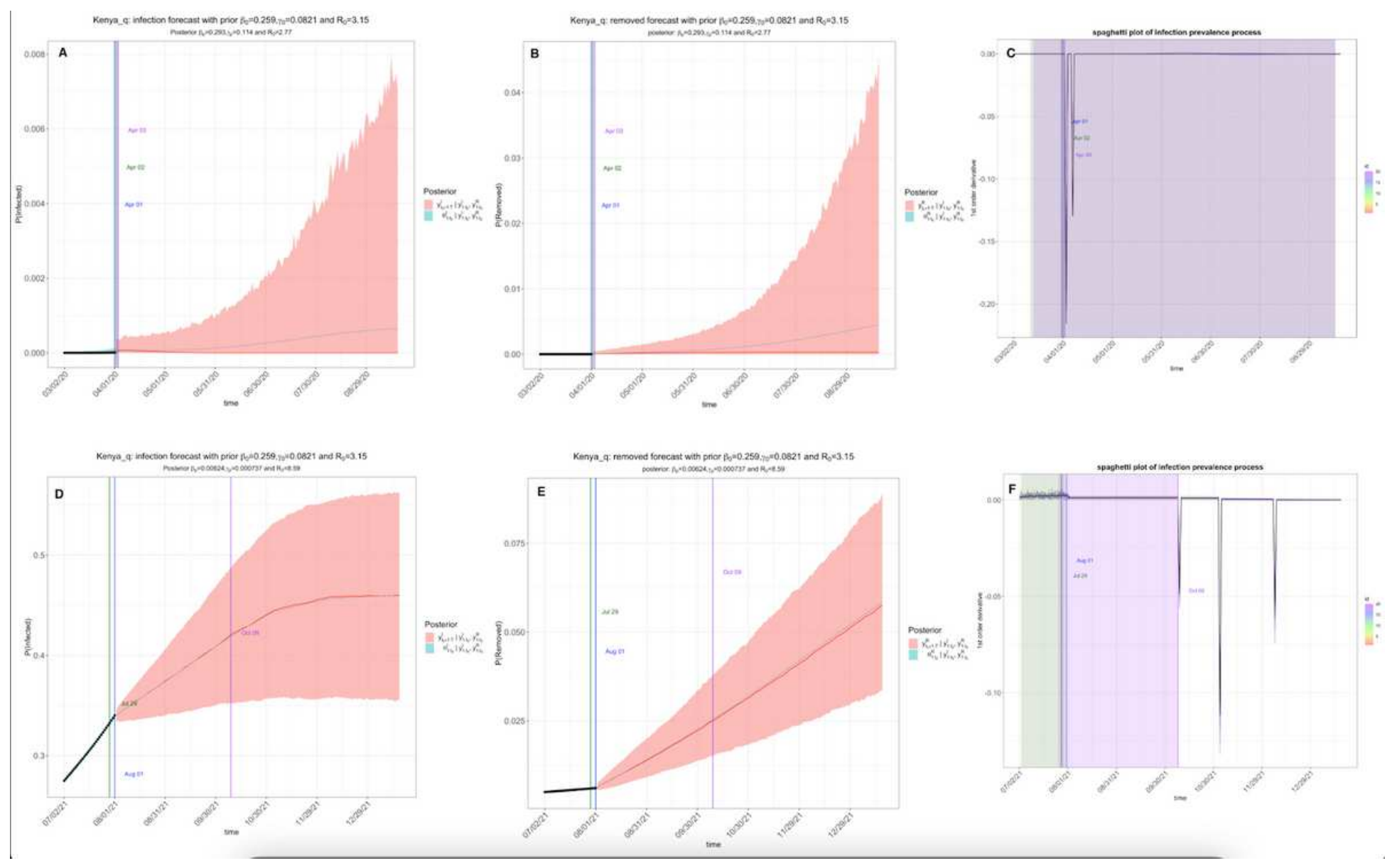

Figure 5

SIR model projection in Kenya with time-varying quarantine. (A) Prediction of COVID-19 infection during 2020/2021 window; (B) Prediction of the removed compartment during 2020/2021 window; (C) Plot of the first-order derivatives of the posterior prevalence of infection in 2020/2021. The colored semitransparent rectangles represent the $95 \% \mathrm{Cl}$ of these turning points. (D) Prediction of the infection of COVID-19 for 2021/2022; (E) Prediction of the removed compartment during 2021/2022 window; (F) Plot of the first-order derivatives of the posterior prevalence of infection. 

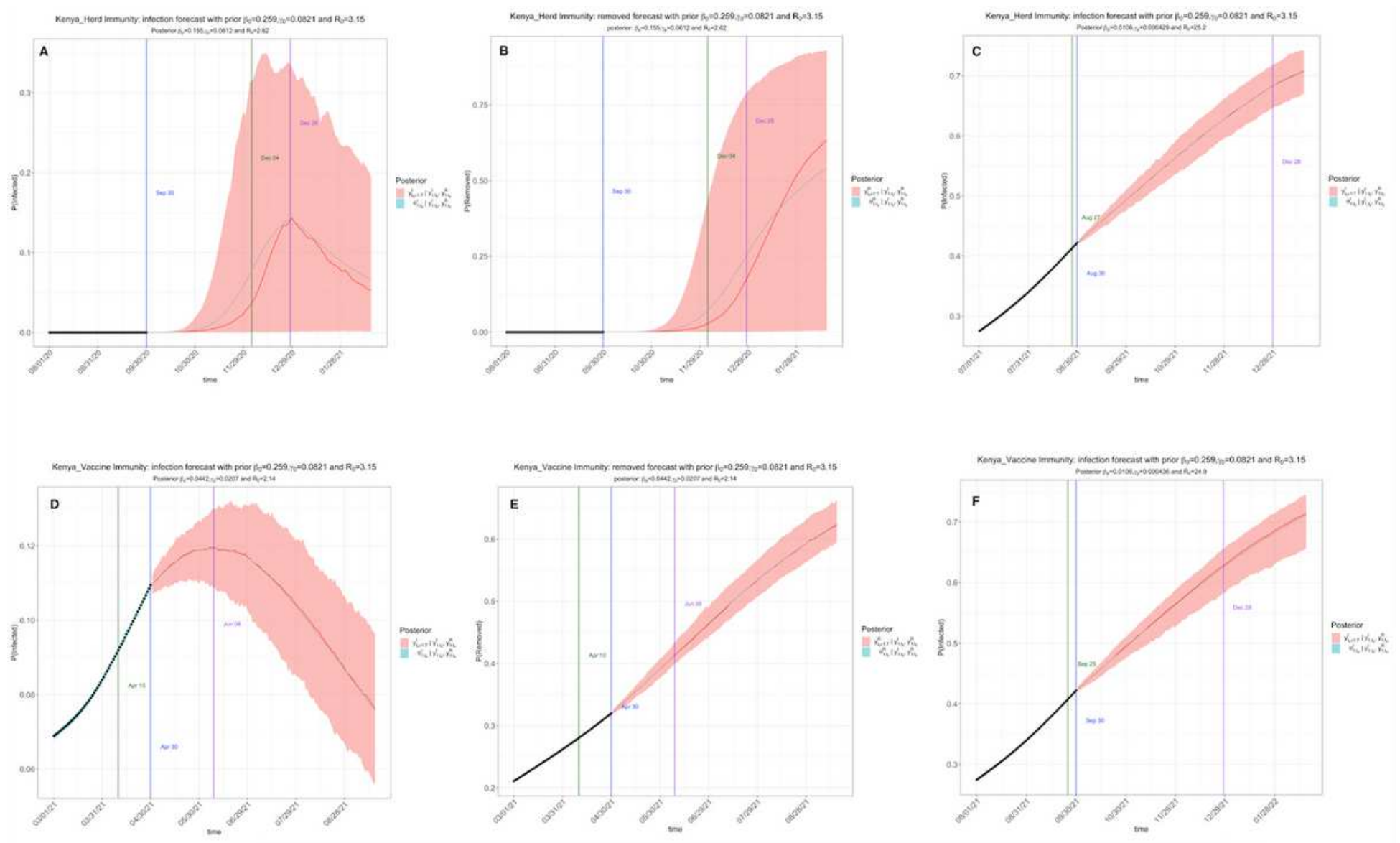

\section{Figure 6}

The extended SIR model simulating herd immunity and vaccination campaign in Kenya. (A) Prediction of COVID-19 infection under the assumption that $20 \%$ of the population has antibodies against SARS-COV2; (B) Prediction of the removed compartment under herd immunity; (C) Projection of the infection in $2021 / 2022$ assuming that $20 \%$ of the population has antibodies against SARS-COV-2; (D) Prediction of COVID-19 infection under the assumption that $2 \%$ of the population is vaccinated; (E) Prediction of the removed compartment assuming that $2 \%$ of the population is vaccinated; (F) Projection of infection in $2021 / 2022$ assuming that $2 \%$ of the population is vaccinated. 


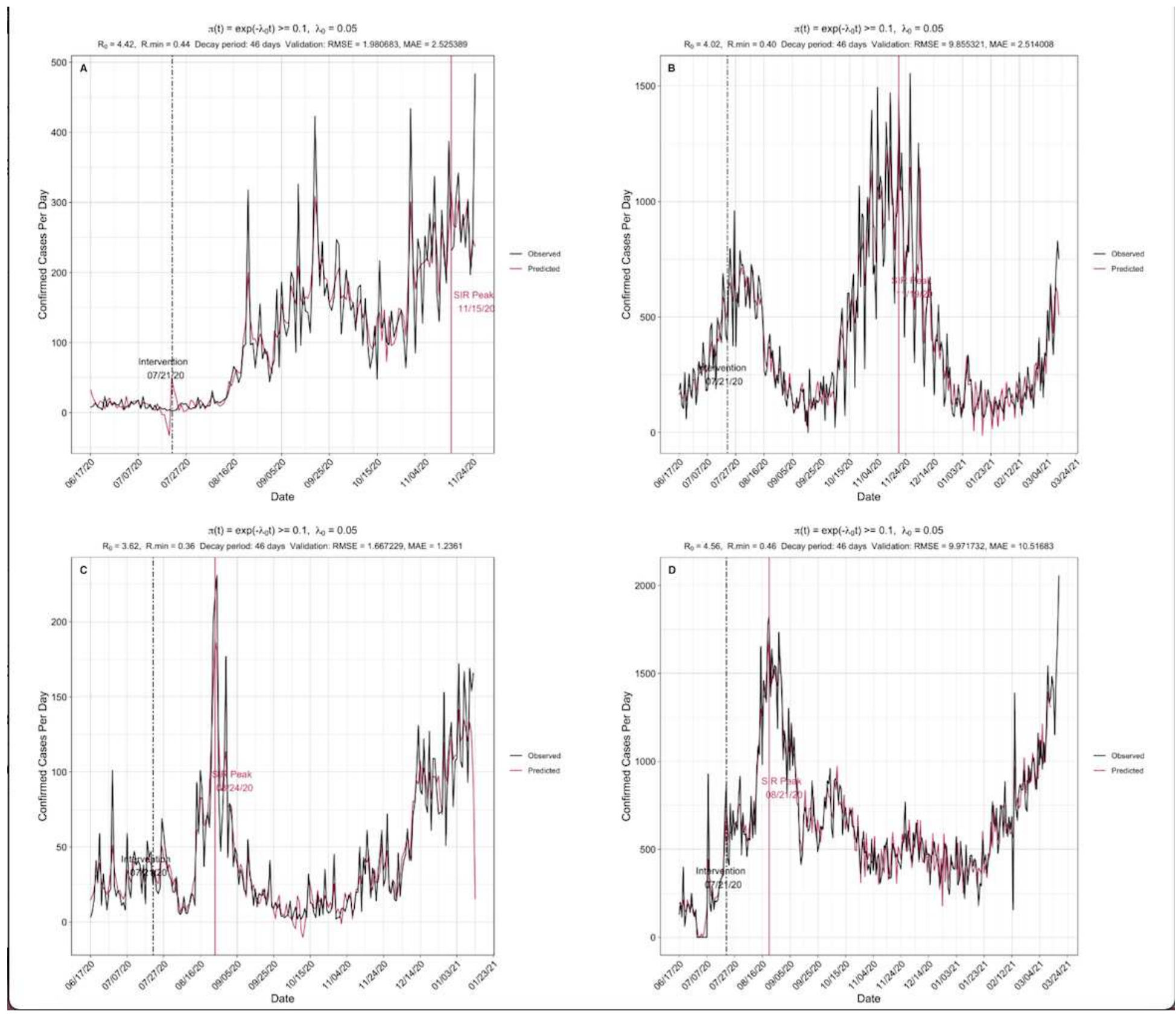

Figure 7

Validation of the model for prediction of infected COVID-19 cases using the Root Mean square error and Mean Absolute Error. The RMSE and MAE values are indicated in the respective figure subtitles. (A) Uganda; (B) Kenya; (C) Rwanda; (D) Ethiopia.

\section{Supplementary Files}

This is a list of supplementary files associated with this preprint. Click to download.

- S1Figure.tif

- S2Figure.tif 
- S3Figure.tif

- S4Figure.tif

- S5Figure.tif

- S6Figure.tif

- S7Figure.tif

- S8Figure.tif

- S9Figure.tif

- S10Figure.tif

- S11Figure.tif

- S12Figure.tif

- S13Figure.tif

- S14Figure.tif

- S15Figure.tif

- S16Figure.tif

- S17Figure.tif

- S18Figure.tif

- S19Figure.tif

- S20Figure.tif

- S21Figure.tif

- S22Figure.tif

- S23Figure.tif

- S24Figure.tif

- S25Figure.tif

- S26Figure.tif

- S27Figure.tif

- S28Figure.tif

- S29Figure.tif

- S30Figure.tif 